\title{
NMR metabolomics for evaluating passage number and harvesting effects on mammalian cell metabolome
}

\begin{abstract}
The variation in the extracellular metabolites of RAW 264.7 cells obtained from different passage numbers (passage 9, 12 and 14) was examined. The impact of different harvesting protocols (trypsinization and scraping) on recovery of intracellular metabolites was then assessed. The similarity and variation in the cell metabolome was investigated using $1 \mathrm{H} \mathrm{NMR}$ metabolic profiling modeled using multivariate data analysis. The characterization and quantification of metabolites was performed to determine the passage-related and harvestingdependent effects on impacted metabolic networks. The trypsinized RAW cells from lower passages gave higher intensities of most identified metabolites, including asparagine, serine and tryptophan. Principal component analysis revealed variation between cells from different passages and harvesting methods, as indicated by the formation of clusters in score plot. Analysis of S-plots revealed metabolites that acted as biomarkers in discriminating cells from different passages including acetate, serine, lactate and choline. Meanwhile lactate, glutamine and pyruvate served as biomarkers for differentiating trypsinized and scraped cells. In passagedependent effects, glycolysis and TCA cycle were influential, whereas glycerophospholipid metabolism was affected by the harvesting method. Overall, it is proposed that typsinized RAW cells from lower passage numbers are more appropriate when conducting experiments related to NMR metabolomics.
\end{abstract}

Keyword: NMR metabolomics; RAW 264.7 cells; Adherent mammalian cells; Cell harvest; Passage number 